\title{
Evidence of a Tonotopic Organization of the Auditory Cortex in Cochlear Implant Users
}

\author{
Jeanne Guiraud ${ }^{1,3,6}$ Julien Besle, ${ }^{2,6}$ Laure Arnold, ${ }^{5}$ Patrick Boyle, ${ }^{5}$ Marie-Hélène Giard, ${ }^{2,6}$ Olivier Bertrand, ${ }^{2,6}$ \\ Arnaud Norena, ${ }^{1,6}$ Eric Truy, ${ }^{1,4,6}$ and Lionel Collet ${ }^{1,3,6}$ \\ ${ }^{1}$ CNRS UMR 5020, Neurosciences and Sensorial Systems Laboratory, Lyon, F-69000, France, and 2INSERM, U821, Lyon, F-69500, France, Departments of \\ ${ }^{3}$ Audiology and Otorhinolaryngology and ${ }^{4}$ Otolaryngology and Head and Neck Surgery, Edouard Herriot University Hospital, Lyon, F-69000, France, \\ ${ }^{5}$ Clinical Research Department, Advanced Bionics, Cambridge CB22 5LD, United Kingdom, and 6 Université Lyon, Lyon, F-69000, France
}

Deprivation from normal sensory input has been shown to alter tonotopic organization of the human auditory cortex. In this context, cochlear implant subjects provide an interesting model in that profound deafness is made partially reversible by the cochlear implant. In restoring afferent activity, cochlear implantation may also reverse some of the central changes related to deafness. The purpose of the present study was to address whether the auditory cortex of cochlear implant subjects is tonotopically organized. The subjects were thirteen adults with at least 3 months of cochlear implant experience. Auditory event-related potentials were recorded in response to electrical stimulation delivered at different intracochlear electrodes. Topographic analysis of the auditory N1 component $(\sim 85$ ms latency) showed that the locations on the scalp and the relative amplitudes of the positive/negative extrema differ according to the stimulated electrode, suggesting that distinct sets of neural sources are activated. Dipole modeling confirmed electrode-dependent orientations of these sources in temporal areas, which can be explained by nearby, but distinct sites of activation in the auditory cortex. Although the cortical organization in cochlear implant users is similar to the tonotopy found in normal-hearing subjects, some differences exist. Nevertheless, a correlation was found between the N1 peak amplitude indexing cortical tonotopy and the values given by the subjects for a pitch scaling task. Hence, the pattern of N1 variation likely reflects how frequencies are coded in the brain.

Key words: cortical tonotopy; cochlear implant; pitch perception; N1 topography; modeling; plasticity

\section{Introduction}

Studies of the N1 component of auditory event-related potentials (ERPs) show that the tonotopic organization exists in the auditory cortex in humans. The N1 response likely results from activation of primary and secondary auditory areas (e.g., Elberling et al., 1982; Näätänen and Picton, 1987). Its topography is characterized by negative potential fields over the frontocentral scalp areas, and positive potentials around the temporal mastoid sites, this polarity reversal being typical of activities in the auditory cortex (Ritter et al., 1972). Several studies have shown that the topography of the N1 component is frequency dependent (Bertrand et al., 1988, 1991; Pantev et al., 1995; Verkindt et al., 1995). Scalp potential distribution changes can be mainly described as a decrease and frontal shift of the frontocentral negativity and an increase of the lateral positivity for higher frequencies. The frequency effect is clearly revealed in the dipole model of N1. The dipole orientation becomes more radial in both coronal and sagittal planes with increasing frequencies. This has been interpreted as the activation of distinct cortical areas in the auditory cortex

Received Jan. 15, 2007; revised May 29, 2007; accepted June 9, 2007.

J.G. was supported by a Convention Industrielle de Formation par la Recherche doctoral grant from Advanced Bionics Europe. We thank our subjects for their kind cooperation.

Correspondence should be addressed to Jeanne Guiraud, Département d'Audiologie et ORL, Pavillon U, Hôpital Edouard Herriot, 5 place d'Arsonval, 69437 Lyon cedex 03, France. E-mail: jeanne guiraud@hotmail.com. DOI:10.1523/JNEUROSCI.0154-07.2007

Copyright $\odot 2007$ Society for Neuroscience $\quad$ 0270-6474/07/277838-09\$15.00/0 with their main orientation following the geometry of the cerebral surface folds. Such an interpretation agrees with the frequency-dependent depth changes of the magnetic N1 (N1m) equivalent source, higher frequencies generating activation of deeper centers (Pantev et al., 1988).

It is likely that the organization of the cortical tonotopic maps is altered with deafness. N1m and psychoacoustical studies showed that cortical neurons deprived of their most sensitive afferent input reorganize to respond to cutoff frequencies in humans (Dietrich et al., 2001; Thai-Van et al., 2002a). Cochlear implant subjects then provide an interesting model in that a profound deafness is made reversible by the implant. Nevertheless, the recovery of afferent inputs delivered by the implant does not necessarily imply a reversion of central changes related to deafness (Irvine, 2000). Moreover, the neural activation resulting from electrical stimulation pulses is very different from neural activation caused by an acoustic stimulation. Importantly, only the more basal part of the cochlea is stimulated by the cochlear implant. As a consequence, if cochlear implantation restores a tonotopic organization, it could be different from the tonotopic organization found in normally hearing subjects. In this context, functional magnetic resonance imaging studies failed to show a cortical tonotopy in cochlear implant users (Melcher et al., 1998; Lazeyras et al., 2002; Seghier et al., 2005). In an ERP study, only one of five subjects showed that activation of different channels resulted in an orderly shift in N1 source location consistent with 
Table 1. Demographic information

\begin{tabular}{|c|c|c|c|c|c|c|}
\hline Subject & Etiology (onset) & Age at test & Duration of deafness (years) & Implant use duration (months) & Speech perception score (\%) & Implanted side \\
\hline S1 & Unknown (progressive) & 16 & 13 & 3 & 60 & Right \\
\hline $\mathrm{S} 2$ & Perinatal asphyxia (sudden) & 17 & 17 & 3 & 45 & Left \\
\hline S3 & Congenital & 20 & 20 & 24 & 28 & Right \\
\hline S4 & Turner syndrome (progressive) & 26 & 2 & 3 & 96 & Left \\
\hline S5 & Unknown (progressive) & 33 & 11 & 9 & 34 & Right \\
\hline S6 & Stroke (sudden) & 50 & 5 & 9 & 76 & Left \\
\hline S7 & Unknown (progressive) & 32 & 1 & 6 & 94 & Right \\
\hline S8 & Genetic (progressive) & 49 & 4 & 3 & 62 & Left \\
\hline \$9 & Chronic otitis (progressive) & 54 & 20 & 6 & 34 & Right \\
\hline $\mathrm{S} 10$ & Meniere's syndrome (progressive) & 65 & 8 & 6 & 42 & Left \\
\hline S11 & Unknown (progressive) & 50 & 12 & 20 & 20 & Right \\
\hline $\mathrm{S} 12$ & Presbycusis (progressive) & 74 & 9 & 6 & 42 & Right \\
\hline $\mathrm{S} 13$ & Unknown (progressive) & 55 & 4 & 6 & 64 & Right \\
\hline
\end{tabular}

Etiology and onset of deafness, age at test, duration of profound bilateral deafness before implantation, duration of cochlear implant use, speech perception score, and side of implantation are shown. Speech perception scores (percentage of phonemes correctly perceived) were obtained at the time of the study using Lafon lists (lists of three phonemes long words) (Lafon, 1964) presented in an open set format in quiet at $65 \mathrm{~dB}$ HL (hearing level) in the sound field.

cochleotopic activation of the human auditory cortex (Ponton et al., 1993).

The primary goal of the present study was to describe the global features of cortical tonotopy in 13 cochlear implant recipients. Topographic analysis and dipole modeling of the N1 component of auditory ERPs were the primary measures used. The study also aimed at comparing this organization with the organization found for normal-hearing subjects and at investigating how the cortical organization relates to the pitch perception by the implant recipients.

\section{Materials and Methods}

Subjects. Thirteen bilaterally profoundly deafened adult subjects ( $10 \mathrm{fe}-$ males) participated in the study (Table 1). They received a HiRes90K cochlear implant (Advanced Bionics Corporation, Sylmar, CA) at the Edouard Herriot University Hospital of Lyon (Lyon, France). Subjects ranged in age from 16 to 74 years. When known, the origin of deafness included perinatal asphyxia $(n=1)$, stroke $(n=1)$, hereditary $(n=1)$, Turner syndrome $(n=1)$, chronic otitis $(n=1)$, Meniere's syndrome $(n=1)$, and presbycusis $(n=1)$. The mean duration of severe-toprofound hearing loss in both ears was 10 years (ranging from 1 to 20 years). All subjects had a full electrode insertion and had at least 3 months of cochlear implant experience at the time of the study, with a mean duration across subjects of 8 months. Five subjects were implanted on the left side, and eight on the right side. All were full-time users of their implants. Benefit from the cochlear implant was variable as revealed by subject speech perception scores to the Lafon lists (Lafon, 1964) (lists of three-phoneme words). Subjects were fully informed of the experimental procedures in accordance with the decision of the local ethical committee. All subjects signed a consent form before participation.

Stimuli. Stimuli were biphasic current pulses of $75 \mu$ s duration, alternated in polarity, and were delivered at a rate of $1.01 \mathrm{~Hz}$. Electrodes 3 (apical), 7, 11, and 15 (basal) were successively stimulated in separate blocks. The chosen stimulation electrodes are separated by $4.4 \mathrm{~mm}$ on the HiFocus $1 j$ electrode array and codes for frequency bands, which center frequencies were 540,1060,2142, and $4264 \mathrm{~Hz}$, respectively. This will allow comparison with the data recorded by Verkindt et al. (1995) in normal-hearing subjects after stimulation with tones of 500, 1000, 2000, and $4000 \mathrm{~Hz}$. Monopolar electrode coupling was selected because this configuration is used in virtually all users' programs. As has been shown (Townshend et al., 1987; Henry et al., 2000), monopolar electrode coupling may increase channel interaction and, thus, transmit spectral information to the cortex less accurately than a bipolar electrode configuration (Bierer and Middlebrooks, 2002; Middlebrooks and Bierer, 2002). However, it is assumed that monopolar electrode coupling can mediate perception of distinct sound frequencies and be used to investigate tonotopy because it yields excellent speech reception (Vandali et al., 2000; Koch et al., 2004; Spahr and Dorman, 2004). A total of 700 electrical stimulation pulses were delivered to each electrode through the Clinical
Programming Interface (Clarion Multistrategy Cochlear Implant System, manual version $2.0,1996)$ connected to the implant system's sound processor. Stimuli were triggered by the SCLIN 2000 fitting software (version 1.08). An intensity rated as comfortably loud by the subject while using a bracketing approach was used for each electrode (Clarion Multistrategy Cochlear Implant System, manual version 2.0, 1996). The presentation order on the various electrodes was counterbalanced across subjects.

ERP recording. Electric potentials were recorded from $30 \mathrm{Ag}-\mathrm{AgCl}$ scalp electrodes referenced to the nose and placed over both hemispheres, according to the 10-20 International System and at additional intermediate locations similar to the montage in Verkindt et al. (1995) for normally hearing subjects. The ground electrode was placed at the forehead. Vertical and horizontal eye movements were monitored using bipolar electrooculogram electrodes placed above the right eye and at its outer canthus. Electrode impedance was $<5 \mathrm{k} \Omega$. EEG was continuously recorded at a sampling rate of $1021 \mathrm{~Hz}$ per channel with a Micromed (Mogliano Veneto, Italy) 98 EEG recording device. The measurements were performed in an electrically shielded room. Because subjects received only computer generated stimuli, their microphone being inactive, the acoustic conditions were not critical. Subjects were seated in a reclining chair and watched a captioned videotape to keep them alert during the measurement session.

EEG analysis was conducted with the ELAN Pack software developed at the Institut National de la Santé et de la Recherche Médicale U821 laboratory (Lyon, France). Evoked responses obtained for each stimulated electrode and each subject were averaged off-line and were baselinecorrected relative to a $100 \mathrm{~ms}$ prestimulus time window. The response to the first stimulus of each run was not included in averaging to avoid contamination by an orienting reflex effect. Automatic artifact rejection excluded signals with excess muscle or eye movement. Epochs with amplitudes exceeding $\pm 100 \mu \mathrm{V}$ were automatically excluded from averaging. However, the automatic rejection mode was switched off in a $10 \mathrm{~ms}$ window around the stimulation pulse for correct interpretation of stimulation artifacts in all responses. Stimulation artifacts were clearly identifiable and reproducible in each trial for electrodes located near the antenna. Amplitudes of three prestimulus and seven poststimulus samples (i.e., $10 \mathrm{~ms}$ ) were thus replaced by linearly interpolated values computed from neighboring samples, to reduce ringing effects in the digital filtering (2-30 Hz; slope, $24 \mathrm{~dB} /$ octave) used to eliminate residual noise.

Topographical analysis. Scalp potential maps were generated using a two-dimensional spherical spline interpolation algorithm (Perrin et al., 1990) and a radial projection from $\mathrm{Cz}$ (top view) or from T3 or T4 (lateral view), thus respecting the length of the meridian arcs. Subjects implanted on the left side had their responses reversed with respect to the midline so that their evoked potentials could be averaged with the responses from subjects implanted on the right side. In some subjects, signals from noisy scalp electrodes near or at the antenna (generally T3 or T4) were replaced by spline interpolated data from the surrounding electrodes.

Latency and amplitude analysis. To analyze the variations of the N1 
component, the relative peak variations of frontal negativity versus temporal positivity was measured as a function of stimulated electrode. The $\mathrm{N} 1$ negative and positive peak latencies and amplitudes were measured in the different stimulus conditions at frontal and temporal subsets of electrodes on each hemiscalp. Contralateral (ipsilateral) frontal subsets included electrodes $\mathrm{FC} 1, \mathrm{Fz}, \mathrm{F} 3, \mathrm{Cz}$, and $\mathrm{C} 3$ (FC2, Fz, F4, Cz, and C4) for right implanted subjects (symmetrical electrode sites for left implanted subjects). Contralateral (ipsilateral) temporal subsets included electrodes OM1, M1, O1, and T5 (OM2, M2, O2, and T6) for right implanted subjects (symmetrical sites for left implanted subjects). The N1 latency was defined as the time to the potential extremum between 50 and $105 \mathrm{~ms}$ after stimulus onset. This latency was measured for each subset of recording electrodes. Such a wide time window was explored because of the high variability of N1 latency in cochlear implantees, something that appears to depend on the duration of deafness (Guiraud et al., 2007), the duration of implant use (Gordon et al., 2004), and the intensity of stimulation (Firszt et al., 2002). The N1 amplitude for each subset of electrodes was then measured as the average amplitude over a $30 \mathrm{~ms}$ time window around the corresponding peak latency.

ANOVAs were performed on the amplitude and latency data, separately for each hemiscalp. In a first approach, frontal and temporal amplitudes and latencies were analyzed separately for each hemisphere using one-way ANOVAs with stimulated electrode as a factor. To reduce intersubject variability of the absolute response amplitude, data were normalized for each subject by the minimum and maximum amplitude values across all four stimulating electrode conditions (i.e., for each subject, the minimum amplitude was set to 0 and the maximum amplitude was set to 1). However, frequency-related changes in amplitude may not necessarily reflect a tonotopic organization (i.e., the activation of different neural populations by different frequencies of stimulation). It could merely be attributable to a general effect of stimulation frequency on the activity of the same neural populations. Therefore, in a second approach, two other ANOVAs were conducted for each hemiscalp to assess genuine tonotopy-related effects. First, assuming an effect of stimulation electrode on N1 amplitude reflecting activation of different neural populations, the absolute values of the frontal and temporal N1 peaks should show the same variation. This hypothesis can be tested with a two-way ANOVA on absolute values of the amplitude with location (frontal vs temporal site) and electrode of stimulation as factors: if there is an interaction between location and stimulation electrode, then the amplitude changes cannot be attributed to an activity modulation of the same neural populations. Second, a tonotopical organization is obviously evidenced if it can be shown that some aspect of the topography of the N1 component changes with the electrode of stimulation. Following Verkindt et al. (1995), we tested for a possible shift of the frontal negativity along a posteroanterior line as a function of stimulation electrode. This was done using two-way ANOVAs on the mean amplitude at two or three frontal electrodes in a $30 \mathrm{~ms}$ time window around the latency of the frontal peak. Electrode location along the anteroposterior axis and electrode of stimulation were factors. A shift would be evidenced by an interaction between electrode location on the scalp and stimulation electrode. Three posteroanterior axes were tested: one on the midline $(\mathrm{Cz}-$ $\mathrm{Fz}$ ), one on the contralateral mediotemporal direction ( $\mathrm{Cz}-\mathrm{FC} 1-\mathrm{F} 3$ for a right-implanted subject), and one on the ipsilateral mediotemporal direction (Cz-FC2-F4 for a right-implanted subject). Data were normalized using the minimum and maximum amplitudes found for each subject. Where appropriate, the Greenhouse-Geisser correction for nonsphericity of data was applied.

Post hoc ANOVAs were also performed to see whether the age, duration of deafness, duration of implant use, and side of implantation (Table 1) have an effect on the cortical organization. Patients were divided into groups according to their age ( $>49$ years old, $n=7 ;<33$ years old, $n=$ 6 ), duration of deafness (more than 10 years, $n=6$; less than 10 years, $n=7$ ), duration of cochlear implant use ( 3 months, $n=4 ; 6$ months, $n=$ 5; $>9$ months, $n=4$ ), and implanted side (left, $n=5$; right, $n=8$ ). The influence of each of these parameters on tonotopy was tested with five three-way ANOVAs with parameters location and electrode of stimulation as factors (ipsilateral frontal shift, contralateral frontal shift, midline frontal shift, ipsilateral frontal/mastoid amplitude balance and contralat- eral frontal/mastoid amplitude balance). A quite liberal threshold of $p=$ 0.15 was chosen because of the small number of patients in each subgroup. However, to limit inflation of type I error, location by frequency interactions for subgroups were only tested when the second-order interaction between the location by frequency interaction and the factor of interest had been significant. All $p$ values were Greenhouse-Geisser corrected, when appropriate.

Dipole source modeling. To further assess the tonotopy of the N1 response's neural sources in the auditory cortex, we considered equivalent current dipoles in a classical three-concentric sphere head model. Conductivity values and radii of the three layers were chosen according to a study by Rush and Driscoll (1969). We have used a time-varying modeling strategy based on dipoles having a stationary location and orientation while their magnitude changes with time (Scherg, 1990). This enabled us to look for one dipole in each hemisphere, corresponding to the simultaneous activity of both auditory cortices within a 50-105 ms time window. Such a model may lead to an oversimplification in the description of the underlying brain activity (Hari, 1990; Lütkenhöner, 2003). Nevertheless, it allows comparison between the different stimulation electrode conditions and comparison with published findings in normal-hearing subjects. Location and orientation parameters for the dipoles were determined by a nonlinear iterative procedure (Marquardt minimization method). Time-varying amplitude was estimated by a least mean square algorithm. The model adequacy was assessed by a goodness-of-fit criterion based on the percentage of experimental variance explained by the model. To estimate 3D confidence intervals on the dipole parameters, we used a Monte Carlo simulation technique as described by Medwick et al. (1989). This consists of adding noise to the potentials created by the dipoles found after a first fitting procedure on the group-averaged data. This latter step is repeated hundreds of time and gives a set of dipole solutions depending on the added noise. The analysis of the threedimensional distribution of this set of dipoles gives an estimate of ellipsoidal confidence intervals around ideal parameter values. The noise added to the theoretical potential values is supposed to represent experimental residual noise. It is a Gaussian noise, digitally filtered between 2 and $30 \mathrm{~Hz}$ (as the experimental data), with a variance equal to the variance of the data in the prestimulus period. Hence, this procedure gave the sensitivity of the best inverse solution to experimental noise. Stationary dipole solutions in the 85-115 ms time window were considered for the grand-averaged $\mathrm{N} 1$ auditory response of normally hearing subjects and based on our four test frequencies. These data were taken from Verkindt et al. (1995) and plotted in the same spherical model as used for our implanted subjects. Dipole orientations and, thus, tonotopic organization of the two populations were compared.

Relationship with perception. Because cochlear implants address mostly the basal part of the cochlea and produce unavoidable current leaks, both frequency-to-place mapping shifts and electrode interactions may occur. This can have considerable effect on pitch perception and cortical organization. Indeed, animal studies of cortical responses to cochlear-implant stimulation have shown substantially broader spatial tuning curves and relatively coarser maps of cochlear place onto cortical surface than what is typical of normal hearing conditions (Merzenich et al., 1977; Snyder et al., 1990, 1991; Raggio and Schreiner, 1999, 2003 Shepherd and Hardie, 2001). After EEG acquisition, subjects were thus required to scale the pitches of pure tones perceived via their normal clinical program. Analog signals (2000 ms duration sinusoids of 500, 1000, 2000, and $4000 \mathrm{~Hz}$ ) generated with an RM1 (Real-time Mini Processor of sound) device (Tucker Davis Technologies, Alachua, FL) were sent into the auxiliary input socket of subjects' processor. First, subjects had to match the loudness of target stimuli $(1000,2000$, or $4000 \mathrm{~Hz})$ to the reference stimulus $(500 \mathrm{~Hz}$, set to comfortably loud level) in a pairedcomparison paradigm. Reference and target stimuli (both of $1 \mathrm{~s}$ duration and separated by $1 \mathrm{~s}$ ) were successively presented. The subject's task was to increase or decrease the level of the target stimulus, according to whether its loudness is softer or louder than that of the target tone, respectively (the level of the reference tone was fixed). The initial level of the target stimulus was set at random between -15 and $+15 \mathrm{~dB}$ around the level of the reference tone. The initial step size, $5 \mathrm{~dB}$, was reduced to $1 \mathrm{~dB}$ after the fourth reversal in signal level. The procedure stopped after 
16 reversals. The equalized level of the target stimulus (used thereafter in the pitch scaling task) was computed as the average signal over the last 12 reversals. Overall, this task ensured that all stimuli in the pitch-scaling task had equivalent loudness. When the loudness-matching procedure was completed, subjects performed the pitch-scaling task. For each sinusoidal stimulation delivered primarily at a given electrode location, subjects had to assign a numerical value in the range of $1-100$, with small and large numbers indicating low and high pitches, respectively. Each stimulus was delivered eight times in a random order. A training block was completed before data collection. Final scores were determined by calculating the mean of the values recorded at each electrode. A one-way ANOVA was used to test whether the mean pitch differed across the four electrode sites. The relationship between N1 peak amplitudes and behavioral measures of pitch perception was evaluated using a Pearson correlation coefficient $(r)$. A group analysis was performed because tonotopic changes of the N1 response were difficult to evidence on an individual basis in a number of subjects, because of poor signal-to-noise ratio.

Post hoc ANOVAs were also performed to see whether the cortical organization found is functional with respect to speech perception. $\mathrm{Pa}-$ tients were divided into two groups according to whether their speechperception scores were $<45 \%(n=7)$ or $>60 \%(n=6)$ of phonemes correctly perceived. These tests were performed in the same way as for post hoc tests involving other parameters from Table 1 (five three-way ANOVAs with speech perception, location, and electrode of stimulation as factors).

\section{Results}

Individual responses revealed an important intersubject variability in N1 latency and topographic changes with stimulation electrode. Thus, whereas single-subject measurements were considered for statistical analysis, a cross-subject grand-average response was also computed at each stimulation electrode to exhibit the major temporal and topographical characteristics of the data.

\section{Topographical analysis}

More stimulation electrodes and more scalp locations were investigated than in the study by Ponton et al. (1993) so as to obtain a more precise characterization of the tonotopic organization. Figure 1 shows the scalp potential maps at the latency of the maximum N1 amplitude at electrode Fz for each stimulation electrode. A polarity reversal between potential fields at frontocentral and temporal scalp areas, which is typical of an activity in the auditory cortex, was observed. Topographical variations of the auditory N1 response with more basal stimulation electrodes were expressed as a decrease of the negativity, which also slightly shifted toward frontal areas, accompanied by an increase of the lateral positivity.

\section{Latency and amplitude analyses}

Figure 2 illustrates the grand-average curves at two frontocentral (FC1; FC2) and temporal (OM1; OM2) sites on each hemiscalp for the four test stimulation electrodes. The polarity reversal between frontal and temporal sites is visible for all stimulation electrodes.

Mean peak latencies and amplitudes and SDs for each stimulation condition for ipsilateral and contralateral frontal and temporal sites are indicated in Table 2. No significant effect of stimulation electrode was found on peak latencies at frontal sites $\left(F_{(3,36)}=0.29 ; p=0.77\right)$ or temporal sites $\left(F_{(3,36)}=0.71 ; p=\right.$ $0.49)$ over the contralateral hemiscalp, nor at the frontal sites $\left(F_{(3,36)}=0.19 ; p=0.78\right)$ or temporal sites $\left(F_{(3,36)}=0.14 ; p=\right.$ 0.87 ) over the ipsilateral hemiscalp. The N1 peak amplitude varies as a monotonic function of electrode of stimulation with a decrease in frontal negativities and increase in temporal positivi-

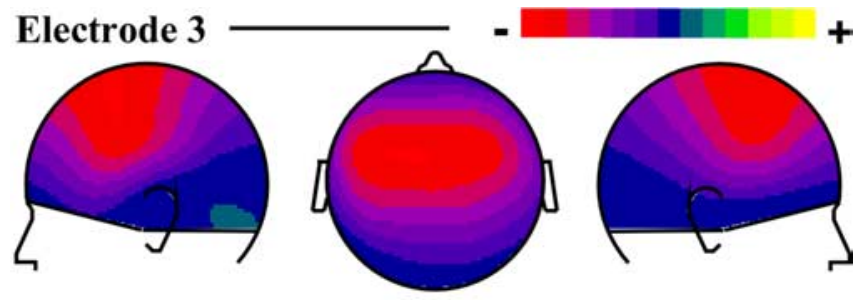

\section{Electrode 7}

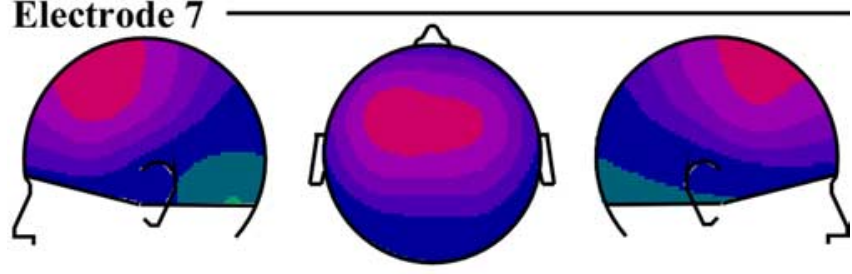

Electrode 11

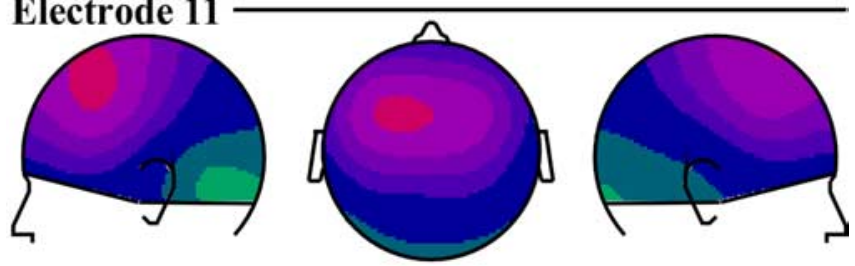

Electrode 15
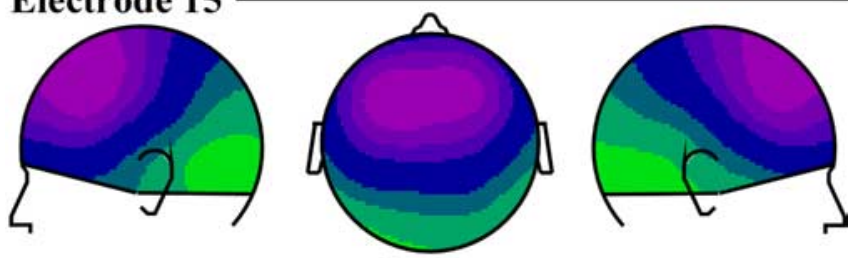

Contralateral view Top view

Ipsilateral view

Figure 1. Scalp potential distributions of the grand-averaged auditory $\mathrm{N} 1$ responses to stimulation at four implant electrode sites (top line, activation of the apical part of the electrode array; bottom line, activation of the basal part of the electrode array). The maps are drawn at the latency of the maximum amplitude at $\mathrm{Fz}(85.5 \mathrm{~ms})$. The topographies are characterized by negative potential fields over frontocentral scalp areas, and positive potentials around the temporal mastoid sites, typical of activities in the auditory cortex. The color scale is $\pm 2.3 \mu \mathrm{V}$. White dots indicate the sites of EEG recording.

ties with more basal stimulation electrode. One-way ANOVAs indicated a significant effect of stimulation electrode at frontocentral sites on the contralateral hemiscalp $\left(F_{(3,36)}=12.81 ; p=\right.$ $0.0002)$, as well as on the ipsilateral hemiscalp $\left(F_{(3,36)}=9.90 ; p=\right.$ $0.0008)$. Although positive amplitudes at mastoids varied similarly with respect to stimulation electrode, no significant effect of stimulation electrode was found for the temporal positivity on the contralateral hemiscalp $\left(F_{(3,36)}=1.46 ; p=0.251\right)$ and it was marginally significant for the ipsilateral hemiscalp $\left(F_{(3,36)}=2.96\right.$; $p=0.068)$. The two-way ANOVAs for absolute peak amplitudes with location (frontal vs temporal site) and stimulation electrode as factors showed significant interactions between scalp location and stimulation electrode on the contralateral side $\left(F_{(3,36)}=7.36\right.$; $p=0.001)$ and on the ipsilateral side $\left(F_{(3,36)}=10.31 ; p=0.0001\right)$. This shows that the joint evolution of the frontal negativity and the temporal positivity as a function of stimulation electrode does not reflect a general effect of stimulation electrode site on the activity of the same neural populations. Two-way ANOVAs with factors of location along the posteroanterior axis and stimulation electrode indicated a significant (location by stimulation electrode) interaction for the ipsilateral hemiscalp along the $\mathrm{Cz} /$ FC2/F4 axis $\left(F_{(6,72)}=3.8 ; p=0.017\right)$, reflecting a shift of the 
frontal negative peak along the posteroanterior, mediolateral axis with more basal stimulation electrode site. No significant frontal shift was found on the midline for $\mathrm{Cz}$ and $\mathrm{Fz}\left(F_{(3,36)}=1.64 ; p=0.21\right)$ and on the contralateral side along the $\mathrm{Cz} / \mathrm{FC1} / \mathrm{F} 3$ axis $\left(F_{(6,72)}=1.6 ; p=0.21\right)$.

Some location by stimulation electrode interactions were also found to interact with parameters from Table 1 . The ipsilateral and contralateral frontal/mastoid joint evolutions were found to interact with the duration of deafness $(p=0.05$ and $p=0.01$, respectively). The joint frontal negativity decrease and temporal positivity increase with more basal stimulation electrode is larger and more significant in subjects with shorter duration of deafness ( $p=0.004$ vs $p=0.09$ ) on the ipsilateral side, whereas it is more significant, but smaller, in subjects with longer duration of deafness $(p=0.001$ vs $p=0.1$ ) on the contralateral side. The contralateral amplitude balance also interacts with the implantation side $(p=0.03)$, the stimulation site effect being monotonically ordered only when subjects are implanted on the right side ( $p=0.01)$. In addition, the central and contralateral frontal shifts with stimulation site were found to interact with the age ( $p=0.11)$, with the shift being significant in younger subjects $(p=$ 0.02 and $p<0.01$, respectively) but not in older subjects. The ipsilateral frontal shift also interacted with duration of implant use $(p=0.08)$ : it was significant only in subjects who have been implanted for 3 months $(p=$ $0.06)$ and $>9$ months $(p=0.01)$.

\section{Grand averaged stationary dipole modeling}

The dipole solutions obtained for the four stimulation electrodes are plotted in Figure 3. Back, top, and lateral two-dimensional views are shown. The $95 \%$ confidence interval ellipsoids for dipole positions show some amount of overlap for the four stimulation electrodes. However, confidence intervals on dipole orientation do not overlap for stimulation electrodes 3,11 , and 15 , as can be seen on the top view in Figure 3. In both hemispheres, the dipole orientation was found to organize orderly with respect to electrode of stimulation. It is tilted anteriorly and medially for more apical stimulation electrodes.

To better compare the tonotopic organization of implanted and normal-hearing subjects, we superimposed our dipole results and the findings from Verkindt et al. (1995). Figure 4 displays top, back, and contralateral views of the dipole solutions for these two populations. Dipolar sources in implant users were found to be more medial than those of normal-hearing subjects. Dipolar sources for electrical stimulation were globally oriented more medially and anteriorly than those obtained for acoustical stimulation. However, the general
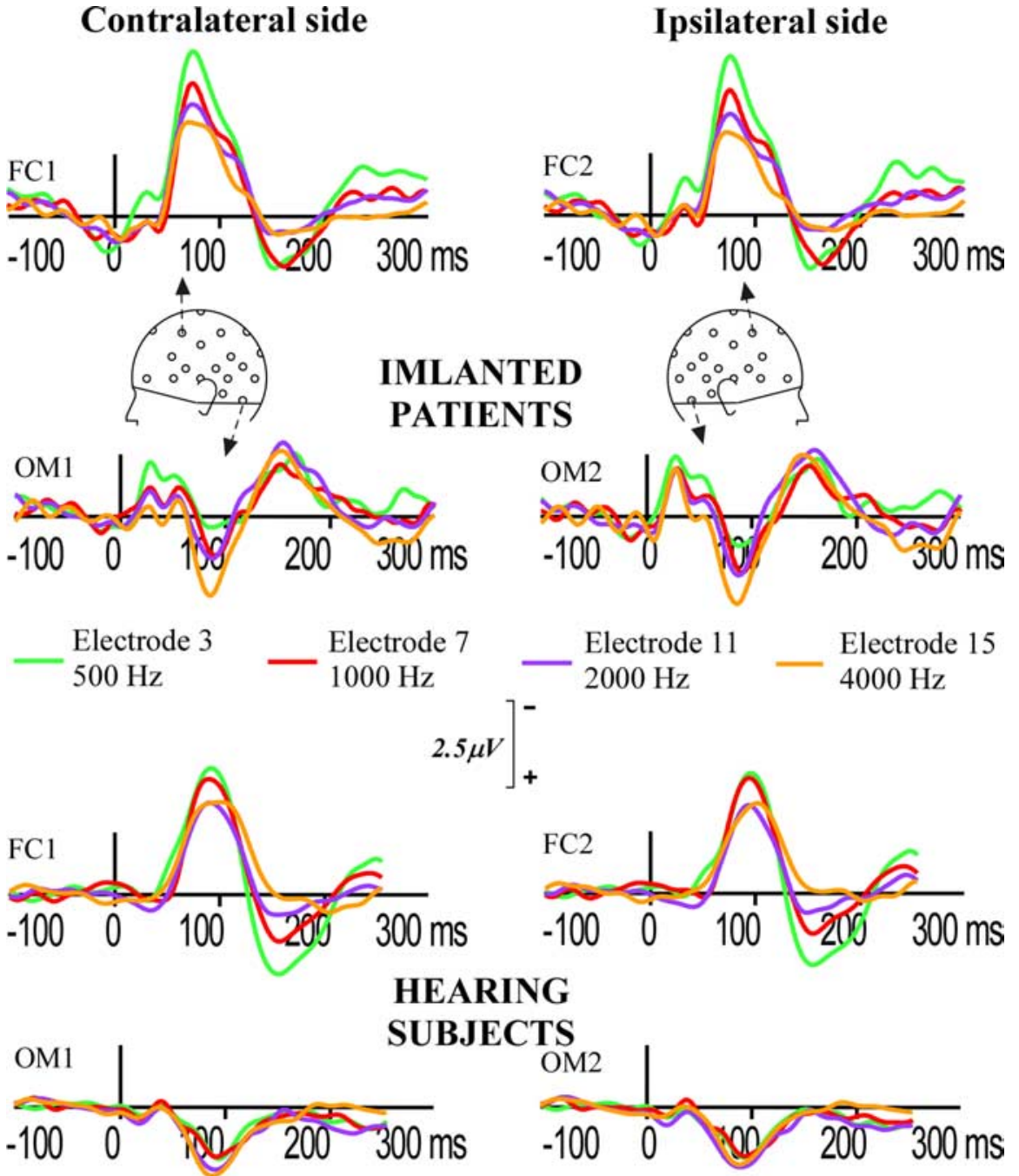

Figure 2. Grand-averaged ERPs at two frontal electrodes (FC1 and FC2), and left (0M1) and right (0M2) mastoidal electrodes for activation of implant electrodes 3 (apical), 7 (mid), 11 (mid), and 15 (basal). FC1 and 0M1 refer to the contralateral hemiscalp and FC2 and $0 M 2$ to the ipsilateral hemiscalp. Negativity is up.

Table 2. Mean peak latencies and amplitudes and SDs for each stimulation condition for ipsilateral and contralateral frontal and temporal sites

\begin{tabular}{|c|c|c|c|c|}
\hline & \multicolumn{2}{|l|}{ Latencies (ms) } & \multicolumn{2}{|l|}{ Amplitude $(\mu \mathrm{V})$} \\
\hline & Ipsilateral side & Contralateral side & Ipsilateral side & Contralateral side \\
\hline \multicolumn{5}{|l|}{ Frontal sites } \\
\hline Electrode 3 & $81 \pm 13$ & $84 \pm 12$ & $-2.59 \pm 1.37$ & $-2.73 \pm 1.36$ \\
\hline Electrode 7 & $84 \pm 12$ & $83 \pm 10$ & $-1.97 \pm 1.29$ & $-2.08 \pm 1.25$ \\
\hline Electrode 11 & $82 \pm 14$ & $82 \pm 14$ & $-1.83 \pm 1.10$ & $-1.90 \pm 1.10$ \\
\hline Electrode 15 & $81 \pm 12$ & $83 \pm 12$ & $-1.61 \pm 1.27$ & $-1.72 \pm 1.31$ \\
\hline \multicolumn{5}{|l|}{ Temporal sites } \\
\hline Electrode 3 & $82 \pm 22$ & $86 \pm 18$ & $0.96 \pm 0.96$ & $1.22 \pm 0.96$ \\
\hline Electrode 7 & $80 \pm 22$ & $80 \pm 21$ & $1.13 \pm 0.97$ & $1.20 \pm 0.80$ \\
\hline Electrode 11 & $78 \pm 20$ & $84 \pm 16$ & $1.13 \pm 1.13$ & $1.21 \pm 1.20$ \\
\hline Electrode 15 & $79 \pm 19$ & $83 \pm 18$ & $1.59 \pm 1.05$ & $1.61 \pm 1.11$ \\
\hline
\end{tabular}

stimulation electrode-dependent tilt is alike with dipoles becoming more medial and anterior with lower frequencies in both populations, especially in the ipsilateral side.

Relationship between perception and cortical tonotopy Because the best marker of cortical tonotopy in cochlear implant recipients was the decrease of the frontal N1 peak amplitude with more basal stimulation, this measure was correlated with pitch- 


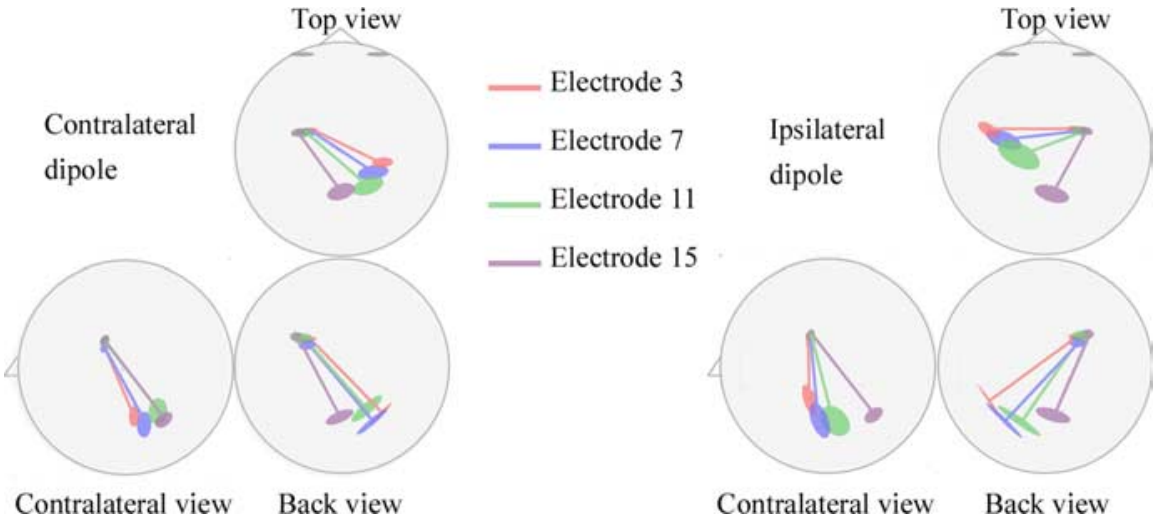

Figure 3. Stationary dipole solutions for the four stimulation electrodes are plotted in back, top, and lateral two-dimensional views. Ninety-five percent confidence interval ellipsoids are drawn around the projections of the dipole positions and orientations.

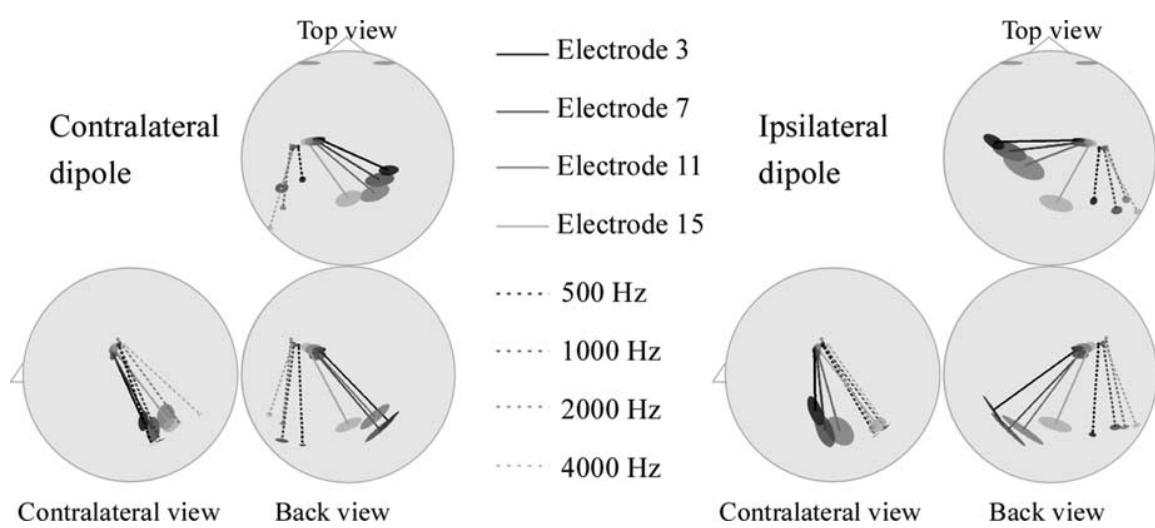

Figure 4. Top, back, and lateral two-dimensional views of stationary dipole solution modeling of the auditory $\mathrm{N} 1$ response to electrical pulses at four stimulation electrodes in cochlear implant users and to four pure tones $(500,1000,2000$, and $4000 \mathrm{~Hz})$ in normal-hearing subjects [data from Verkindt et al. (1995)]. Ninety-five percent confidence interval ellipsoids are drawn around the projections of the dipole positions and orientations.

scaling data. The monotonic increase in pitch estimates from the apical to the basal end of the intracochlear electrode array (low to high numbered electrodes) in Figure $5 a$ is indicative of a tonotopic pitch structure. A Kruskal-Wallis test showed that subjects attributed significantly different pitch values to the various stimulation electrodes $(p<0.001)$. The Pearson test showed a highly significant inverse correlation between the frontal N1 peak amplitude and the pitch values reported by the subjects $(r=-0.99$; $p<0.001$ ), as illustrated in Figure $5 b$.

Moreover, the tonotopic organization of ERPs seems to be related to the ability of the patient to perceive speech: there were marginally significant second-order interactions between speech perception score and the frontal shift of the $\mathrm{N} 1$ peak on both ipsilateral and contralateral sides of the scalp $(p=0.10$ and $p=$ 0.12 , respectively), and on the midline $(p=0.15)$. In all cases, a significant tonotopy effect was evidenced only in the subgroup with better scores (ipsilateral side, $p=0.02$; contralateral side, $p=0.04$; midline, $p=0.09$ ).

\section{Discussion}

Evidence of a tonotopic organization of the auditory cortex in cochlear implant recipients

For all electrodes of stimulation examined, the N1 topography of auditory ERPs in cochlear implant users was characterized by a negative potential field over frontocentral areas associated with positive potentials around the temporal mastoid sites. This was a scalp distribution typical of activity in the auditory cortex (Ritter et al., 1972). Moreover, we found that moving stimulation from apical to more basal sites of the cochlea resulted in a shift of the N1 frontocentral negativity toward anterior sites. Such change was associated with alterations in the ratio of frontal (negative)/temporal (positive) potential amplitudes. This resulted in a negativity decrease accompanied by a positivity increase on topographic maps. These observations are similar to the tonotopic variations of the auditory N1 component in response to acoustic stimuli from low to high frequencies in normal-hearing subjects (Verkindt et al., 1995). Altogether, our results strongly suggest that, after at least 3 months of cochlear implant use, the recipient's auditory cortex presents a tonotopic organization that resembles the frequency maps of normal-hearing subjects. This could be shown despite the fact that individual potential maps presented a great intersubject variability, probably because of the diversity in the size and geometry of the cortex in the Sylvian fissure (Larsen et al., 1989), as well as in demographics, and deafness and implantation characteristics. Even if more subjects would be necessary to assert this, it seems that the tonotopy effects are better defined and more significant for younger patients and with a shorter history of deafness. The $\mathrm{N} 1$ topographic changes with the electrode of stimulation were observed over both hemiscalps, but were more significant over the ipsilateral hemisphere when all data were considered and more significant over the left hemisphere in subjects implanted on the right side. This observation was, however, difficult to interpret because, in normal-hearing subjects, it is unclear whether the tonotopic maps of the auditory cortex are similar for ipsilateral and contralateral or left and right stimulation (Verkindt et al., 1995; Strainer et al., 1997; Pantev et al., 1998; Liegeois-Chauvel et al., 2001; Gabriel et al., 2004).

As in Bertrand et al. (1991) and Verkindt et al. (1995), a simple dipolar model was considered to describe the major tonotopic features. This was done essentially in terms of dipole orientations for it is known that electrical recording allows more accurate estimation of dipole orientations than dipole locations (Stok et al., 1987). As seen in Figure 3, rather vertically oriented dipoles were found to explain the N1 wave generated by activation of each electrode. The resultant orientations of this activation follow the geometry of the folded outer surface of the cortex. The stimulation site effect is clearly revealed by the dipole orientation because three of four orientation confidence intervals did not overlap in either hemisphere. This suggests that closely spaced, yet different, cortical areas are activated. Confidence intervals on dipole orientations that correspond to electrodes 3,7 , and 11 overlap more than for electrode 15 . This fact could be explained by the electrode array addressing mostly the basal part of the cochlea. Blamey et al. (1996) found that higher acoustically initiated frequencies tend to be matched by more basal electrodes in the opposite ear. The tonotopy should therefore get reorganized 

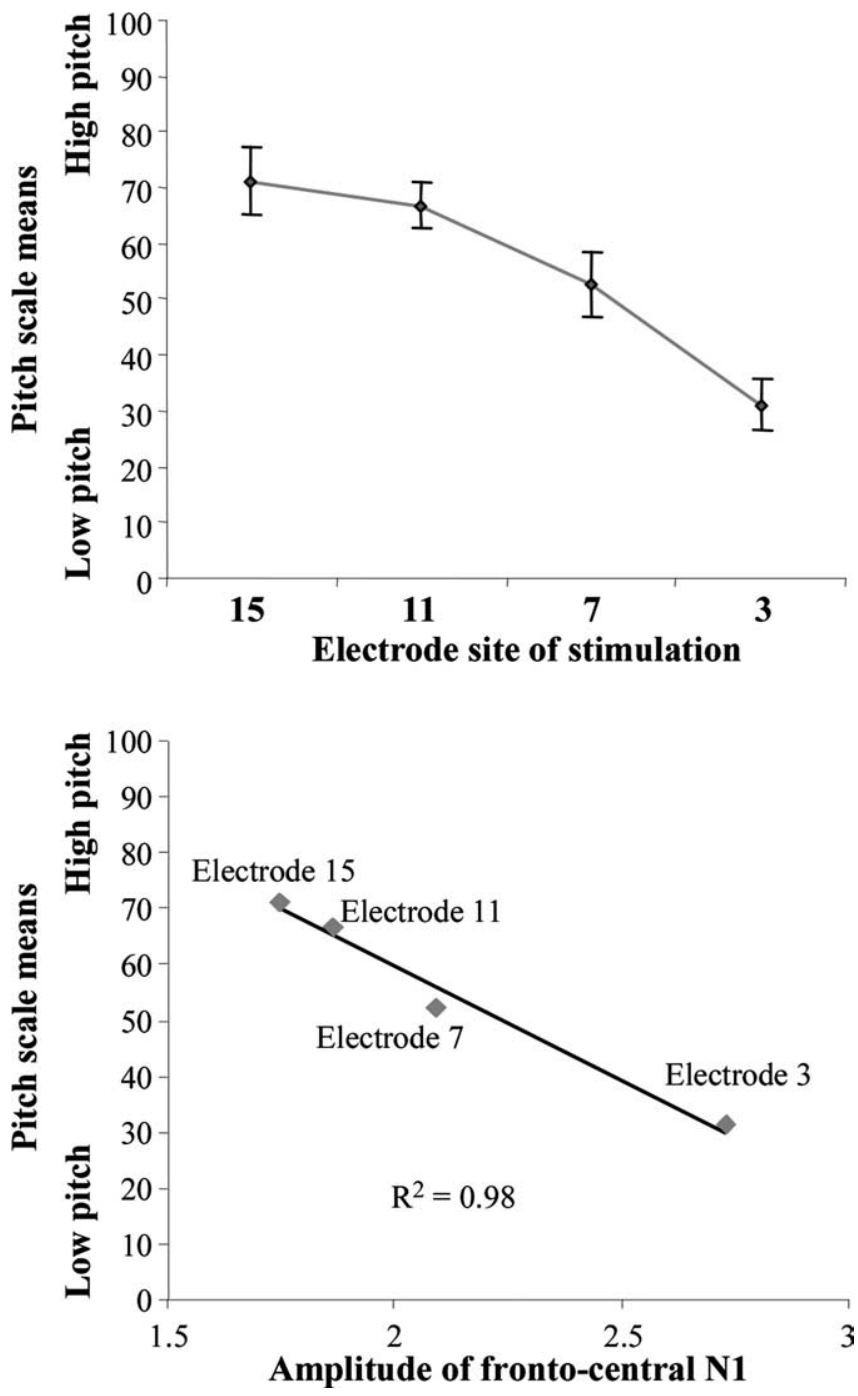

Figure 5. Top, Grand-averaged data for the pitch scaling task. Stimulation electrodes are plotted along the abscissa. Vertical bars indicate the SE around each mean. Bottom, Scatterplots show the relationship between the mean values of the pitch scale test and the grand-averaged measures of the maximal frontocentral $\mathrm{N} 1$ amplitude for electrodes 3, 7, 11, and 15.

easily for high frequencies (e.g., for activation of electrode 15). However, Dorman et al. (1994) have shown that the electrically initiated frequencies on the most apical electrodes are slightly higher than the acoustic frequencies that these electrodes are programmed to code. This may result in a more confused representation of lower frequencies at the cortical level and explain why dipoles orientations are less well separated for activation of electrodes 3, 7, and 11 in Figure 4. However, the dipole orientation becomes more anterior, inferior, and medial with more apical electrodes and resembles the orientation shifts in normal-hearing subjects. It is therefore likely that the generators that are activated by electrical stimulation are roughly organized the same way as in normal-hearing subjects. This orientation shift may represent that higher frequencies activate deeper generators as demonstrated by Bertrand et al. (1991).

Inferring precise tonotopic organization from dipole orientation is speculative. The topographic changes observed may indeed reflect the resultant of the several different tonotopic maps that have been shown to exist in the human auditory cortex (Pantev et al., 1995; Formisano et al., 2003; Talavage et al., 2004). Gabriel et al. (2004) proposed two hypotheses to explain varia- tions of a single dipolar source in the auditory cortex. According to the first one, N1 would be attributable to one predominant generator and a few secondary activation areas, the dipolar source then reflecting one major tonotopic map. The second hypothesis is that N1 would result from the activation of several frequencydependent areas of more or less equal importance, the dipole variation reflecting a general tonotopic trend without specific physiological reality. In any case, our findings provide evidence for a global tonotopic organization of the auditory cortex in cochlear implant recipients. This cortical tonotopy either already existed or, if deafness had altered the pre-existing maps, may result from cortical plasticity reorganization because of auditory rehabilitation after at least 3 months of implant experience.

\section{Additional comparison with normal-hearing subjects}

Figure 4 showed that the dipolar sources modeling the N1 component in cochlear implantees are located and oriented more anteriorly and medially than those found in normal-hearing subjects. This may suggest that different parts of the auditory cortex with different orientations are activated in these two populations. Ponton et al. (1993) also noted a lateral shift in source location, which they thought might be caused by contamination of the scalp wave forms by electrical artifact from the implant, or the activation of nonauditory (e.g., somatosensory) structures. However, this can hardly account for the differences observed in the present study because source locations were equally shifted in both hemispheres in implantees, despite the fact that the artifact occurred only around the antenna on one side of the head. In addition, none of the subjects reported facial nerve activation contrary to the subjects in the study by Ponton et al. (1993).

Direct comparison between the locations/orientations of the $\mathrm{N} 1$ dipolar sources in cochlear implantees and in the subjects in the study by Verkindt et al. (1995) should be made with caution. Indeed, despite similar measurement conditions, differences between electrical and acoustical stimulations and, consequently, in data analysis, could not be avoided. This may contribute to the differences observed between implanted and normal-hearing subjects. For instance, the use of shorter stimuli in implantees could lead to the location difference observed. Rosburg et al. (2002) have shown that the shorter the stimulus, the more anterior and inferior the dipole location of N1m. The longer time window of N1 analysis in implantees' data (55-105 ms) than in the Verkindt et al. (1995) study (85-115 ms) may also have resulted in the inclusion of additional earlier latency generators into the modeling procedure. This explanation is, however, unlikely for several reasons. First, it is known that electrically evoked potentials occur earlier than acoustically evoked potentials (Ponton and Don, 1995). Two factors may contribute to this latency shortening: the delay in the traveling acoustic wave and the transduction delay are bypassed in cochlear implantees, and electrical current pulses result in much better synchronization of a larger number of neural units. Second, if it had been the case, the tonotopic maps of the middle latency responses and of the N1 component, which mirror each other (Pantev et al., 1995), would have at least partly cancelled one another out. This does not appear to be occurring here. The fact that the tonotopic distribution observed varies similarly to that of the N1 component in normalhearing subjects would rather indicate that the same stage of frequency processing was being investigated.

\section{Relationship with perception}

The subjects were able to discriminate and appropriately scale the pitches generated by activation of the electrodes used to obtain 
the cortical mapping. As illustrated in Figure 5, their ability to scale higher pitches correlated with the decrease in the cortical frontocentral negativity, which was found to be a characteristic of the tonotopic organization of the auditory cortex. Hence, it is reasonable to propose that the cortical neuronal frequency selectivity at the physiological level contributes to frequency discrimination at the perceptual level. The ability to extract pitch from sounds being a fundamental attribute of hearing, the possible cortical reorganization of tonotopy with implant use may have clinical implications. Indeed, in cochlear implant recipients, better performance in electrode discrimination correlates with improvements in speech perception (Busby et al., 1993; Donaldson and Nelson, 2000; Henry et al., 2000) and, when frequency allocation in the cochlea does not respect the frequency-place mapping, speech perception is poorer (Friesen et al., 1999). This is confirmed by the fact that subjects with the best speech perception scores present better defined and more significant tonotopic $\mathrm{N} 1$ effects than subjects with poorer scores. Cochlear implant benefit may therefore depend on the way cortical tonotopic maps are organized and adapted to electrical stimulation of the basal part of the cochlea. A study similar to the present study should be conducted over time from implant switch on to investigate the plastic aspects of the tonotopic maps.

\section{References}

Bertrand O, Perrin F, Echallier JF and Pernier J (1988) Topography and model analysis of auditory evoked potentials: tonotopic aspects. In: Functional brain imaging (Pfurtscheller G, Lopes da Silva FH, eds) pp 75-82. Toronto: Hans Huber.

Bertrand O, Perrin F, Pernier J (1991) Evidence for a tonotopic organization of the auditory cortex observed with auditory evoked potentials. Acta Otolaryngol [Suppl] 491:116-122; discussion 123.

Bierer JA, Middlebrooks JC (2002) Auditory cortical images of cochlearimplant stimuli: dependence on electrode configuration. J Neurophysiol 87:478-492.

Blamey PJ, Dooley GJ, Parisi ES, Clark GM (1996) Pitch comparisons of acoustically and electrically evoked auditory sensations. Hear Res 99:139-150.

Busby PA, Tong YC, Clark GM (1993) Electrode position, repetition rate, and speech perception by early- and late-deafened cochlear implant patients. J Acoust Soc Am 93:1058-1067.

Dietrich V, Nieschalk M, Stoll W, Rajan R, Pantev C (2001) Cortical reorganization in patients with high frequency cochlear hearing loss. Hear Res 158:95-101.

Donaldson GS, Nelson DA (2000) Place-pitch sensitivity and its relation to consonant recognition by cochlear implant listeners using the MPEAK and SPEAK speech processing strategies. J Acoust Soc Am 107:1645-1658.

Dorman MF, Smith M, Smith L, Parkin JL (1994) The pitch of electrically presented sinusoids. J Acoust Soc Am 95:1677-1679.

Elberling C, Bak C, Kofoed B, Lebech J, Saermark K (1982) Auditory magnetic fields from the human cerebral cortex: location and strength of an equivalent current dipole. Acta Neurol Scand 65:553-569.

Firszt JB, Chambers RD, Kraus, Reeder RM (2002) Neurophysiology of cochlear implant users I: effects of stimulus current level and electrode site on the electrical ABR, MLR, and N1-P2 response. Ear Hear 23:502-515.

Formisano E, Kim DS, Di Salle F, van de Moortele PF, Ugurbil K, Goebel R (2003) Mirror-symmetric tonotopic maps in human primary auditory cortex. Neuron 40:859-869.

Friesen LM, Shannon RV, Slattery III WH (1999) The effect of frequency allocation on phoneme recognition with the nucleus 22 cochlear implant. Am J Otol 20:729-734.

Gabriel D, Veuillet E, Ragot R, Schwartz D, Ducorps A, Norena A, Durrant JD, Bonmartin A, Cotton F, Collet L (2004) Effect of stimulus frequency and stimulation site on the $\mathrm{N} 1 \mathrm{~m}$ response of the human auditory cortex. Hear Res 197:55-64.

Gordon KA, Papsin BC, Harrison RV (2004) Toward a battery of behavioral and objective measures to achieve optimal cochlear implant stimulation levels in children. Ear Hear 25:447-463.

Guiraud J, Gallego S, Arnold L, Boyle P, Truy E, Collet L (2007) Effects of auditory pathway anatomy and deafness characteristics? Part 2: On electrically evoked late auditory responses. Hear Res, in press.

Hari R (1990) Magnetic evoked fields of the human brain: basic principles and applications. Electroencephalogr Clin Neurophysiol [Suppl] 41:3-12.

Henry BA, McKay CM, McDermott HJ, Clark GM (2000) The relationship between speech perception and electrode discrimination in cochlear implantees. J Acoust Soc Am 108:1269-1280.

Irvine DR (2000) Injury- and use-related plasticity in the adult auditory system. J Commun Disord 33:293-311; quiz 311-292.

Koch DB, Osberger MJ, Segel P, Kessler D (2004) HiResolution and conventional sound processing in the HiResolution bionic ear: using appropriate outcome measures to assess speech recognition ability. Audiol Neurootol 9:214-223.

Lafon JC (1964) Le test phonétique et la mesure de l'audition. Eindhoven: Centrex.

Larsen JP, Odegaard H, Grude TH, Hoien T (1989) Magnetic resonance imaging - a method of studying the size and asymmetry of the planum temporale. Acta Neurol Scand 80:438-443.

Lazeyras F, Boex C, Sigrist A, Seghier ML, Cosendai G, Terrier F, Pelizzone M (2002) Functional MRI of auditory cortex activated by multisite electrical stimulation of the cochlea. NeuroImage 17:1010-1017.

Liegeois-Chauvel C, Giraud K, Badier JM, Marquis P, Chauvel P (2001) Intracerebral evoked potentials in pitch perception reveal a functional asymmetry of the human auditory cortex. Ann NY Acad Sci 930:117-132.

Lütkenhöner B (2003) Magnetoencephalography and its Achilles' heel. J Physiol (Paris) 97:641-658.

Medwick PA, Lewis PS, Aine C, Flynn FR (1989) Monte Carlo analysis of localization errors in magnetoencephalography. In: Advances in Biomagnetism (Williamson SJ, Hoke M, Stroink G, eds), pp 543-546. New York: Plenum.

Melcher JR, Eddington DK, Gracia N, Qin M, Sroka J, Weisskoff RM (1998) Electrically evoked cortical activity in cochlear implant subjects can be mapped using fMRI. NeuroImage 7:S385.

Merzenich MM, White MW, Leake PA, Schindler RA, Michelson RP (1977) Further progress in the development of multichannel cochlear implants. Trans Sect Otolaryngol Am Acad Ophthalmol Otolaryngol 84:181-182.

Middlebrooks JC, Bierer JA (2002) Auditory cortical images of cochlearimplant stimuli: coding of stimulus channel and current level. J Neurophysiol 87:493-507.

Näätänen R, Picton T (1987) The N1 wave of the human electric and magnetic response to sound: a review and an analysis of the component structure. Psychophysiology 24:375-425.

Pantev C, Hoke M, Lehnertz K, Lutkenhoner B, Anogianakis G, Wittkowski W (1988) Tonotopic organization of the human auditory cortex revealed by transient auditory evoked magnetic fields. Electroencephalogr Clin Neurophysiol 69:160-170.

Pantev C, Bertrand O, Eulitz C, Verkindt C, Hampson S, Schuierer G, Elbert $\mathrm{T}$ (1995) Specific tonotopic organizations of different areas of the human auditory cortex revealed by simultaneous magnetic and electric recordings. Electroencephalogr Clin Neurophysiol 94:26-40.

Pantev C, Ross B, Berg P, Elbert T, Rockstroh B (1998) Study of the human auditory cortices using a whole-head magnetometer: left vs right hemisphere and ipsilateral vs contralateral stimulation. Audiol Neurootol 3:183-190.

Perrin F, Bertrand O, Giard MH, Pernier J (1990) Precautions in topographic mapping and in evoked potential map reading. J Clin Neurophysiol 7:498-506.

Ponton CW, Don M (1995) The mismatch negativity in cochlear implant users. Ear Hear 16:131-146.

Ponton CW, Don M, Waring MD, Eggermont JJ, Masuda A (1993) Spatio-temporal source modeling of evoked potentials to acoustic and cochlear implant stimulation. Electroencephalogr Clin Neurophysiol 88:478-493.

Raggio MW, Schreiner CE (1999) Neuronal responses in cat primary auditory cortex to electrical cochlear stimulation. III. Activation patterns in short- and long-term deafness. J Neurophysiol 82:3506-3526.

Raggio MW, Schreiner CE (2003) Neuronal responses in cat primary auditory cortex to electrical cochlear stimulation: IV. Activation pattern for sinusoidal stimulation. J Neurophysiol 89:3190-3204.

Ritter M, Simson R, Vaughan HG (1972) Association cortex potentials and 
reaction time in auditory discrimination. Electroencephalogr Clin Neurophysiol 33:547-555.

Rosburg T, Haueisen J, Sauer H (2002) Stimulus duration influences the dipole location shift within the auditory evoked field component N100m. Brain Topogr 15:37-41.

Rush S, Driscoll DA (1969) EEG electrode sensitivity-an application of reciprocity. IEEE Trans Biomed Eng 16:15-22.

Scherg M (1990) Fundamentals of dipole source potential analysis. In: Auditory evoked magnetic fields and electrical potentials. Advances in audiology (Grandori F, Hoke M, Romani GL, eds), pp 40-69. Basel: Karger.

Seghier ML, Boex C, Lazeyras F, Sigrist A, Pelizzone M (2005) FMRI evidence for activation of multiple cortical regions in the primary auditory cortex of deaf subjects users of multichannel cochlear implants. Cereb Cortex 15:40-48.

Shepherd RK, Hardie NA (2001) Deafness-induced changes in the auditory pathway: implications for cochlear implants. Audiol Neurootol 6:305-318.

Snyder RL, Rebscher SJ, Cao KL, Leake PA, Kelly K (1990) Chronic intracochlear electrical stimulation in the neonatally deafened cat. I: Expansion of central representation. Hear Res 50:7-33.

Snyder RL, Rebscher SJ, Leake PA, Kelly K, Cao K (1991) Chronic intracochlear electrical stimulation in the neonatally deafened cat. II. Temporal properties of neurons in the inferior colliculus. Hear Res 56:246-264.

Spahr AJ, Dorman MF (2004) Performance of subjects fit with the Ad- vanced Bionics CII and Nucleus 3G cochlear implant devices. Arch Otolaryngol Head Neck Surg 130:624-628.

Stok CJ, Meijs JW, Peters MJ (1987) Inverse solutions based on MEG and EEG applied to volume conductor analysis. Phys Med Biol 32:99-104.

Strainer JC, Ulmer JL, Yetkin FZ, Haughton VM, Daniels DL, Millen SJ. (1997) Functional MR of the primary auditory cortex: an analysis of pure tone activation and tone discrimination. AJNR Am J Neuroradiol 18:601-610.

Talavage TM, Sereno MI, Melcher JR, Ledden PJ, Rosen BR, Dale AM (2004) Tonotopic organization in human auditory cortex revealed by progressions of frequency sensitivity. J Neurophysiol 91:1282-1296.

Thai-Van H, Micheyl C, Norena A, Collet L (2002a) Local improvement in auditory frequency discrimination is associated with hearing-loss slope in subjects with cochlear damage. Brain 125:524-537.

Townshend B, Cotter N, Van Compernolle D, White RL (1987) Pitch perception by cochlear implant subjects. J Acoust Soc Am 82:106-115.

Vandali AE, Whitford LA, Plant KL, Clark GM (2000) Speech perception as a function of electrical stimulation rate: using the Nucleus 24 cochlear implant system. Ear Hear 21:608-624.

Verkindt C, Bertrand O, Perrin F, Echallier JF, Pernier J (1995) Tonotopic organization of the human auditory cortex: N100 topography and multiple dipole model analysis. Electroencephalogr Clin Neurophysiol 96:143-156. 